\title{
RADIO IMAGING OF THE NGC 1333 IRAS 4B REGION
}

\author{
Minho Choi ${ }^{1,2}$ And JeOng-Eun LEE ${ }^{3}$ \\ ${ }^{1}$ Korea Astronomy and Space Science Institute, 776 Daedeokdaero, Yuseong, Daejeon 305-348, Korea \\ E-mail:minho@kasi.re.kr \\ 2 University of Science and Technology, 217 Gajeongro, Yuseong, Daejeon 305-350, Korea \\ 3 Department of Astronomy and Space Science, Kyung Hee University, Yongin, Gyeonggi 446-701, Korea \\ (Received August 24, 2011; Revised September 21, 2011; Accepted September 23, 2011)
}

\begin{abstract}
The NGC 1333 IRAS 4B region is observed in the $6.9 \mathrm{~mm}$ and $1.3 \mathrm{~cm}$ continuum with an angular resolution of about 0.4 arcseconds. IRAS $4 \mathrm{BI}$ is detected in both bands, and BII is detected in the 6.9 $\mathrm{mm}$ continuum only. The $1.3 \mathrm{~cm}$ source of BI seems to be a disk-like flattened structure with a size of about 50 AU. IRAS 4BI does not show any sign of multiplicity. Examinations of archival infrared images show that the dominating emission feature in this region is a bright peak in the southern outflow driven by BI, corresponding to the molecular hydrogen emission source HL 9a. Both BI and BII are undetectable in the mid-IR bands. The upper limit on the far-IR flux of IRAS 4BII suggests that it may be a very low luminosity young stellar object.
\end{abstract}

Key words : ISM: individual (NGC 1333 IRAS 4B) — ISM: structure — stars: formation

\section{INTRODUCTION}

NGC 1333 IRAS 4B is a Class 0 young stellar object in Perseus molecular cloud at a distance of 235 pc from the Sun (Sandell et al. 1991; Hirota et al. 2008; Enoch et al. 2009). Radio and infrared observations revealed various star forming activities in this region, such as molecular outflows and $\mathrm{H}_{2} \mathrm{O}$ masers (Blake et al. 1995; Hodapp \& Ladd 1995; Choi et al. 1999, 2004, 2006; Rodríguez et al. 1999, 2002; Choi 2001; Reipurth et al. 2002; Park \& Choi 2007). Interferometric observations revealed that there are two compact continuum sources, BI and BII, with an angular separation of $11^{\prime \prime}$ (Looney et al. 2000; Choi 2001). BI is a protostellar object, and most of the star forming activities in this region can be attributed to BI. By contrast, BII has been detected in the millimeter and submillimeter wavelength bands only, and its nature is less clear.

Lay et al. (1995) performed a single-baseline submillimeter interferometry with a resolution of $\sim 0 . .5$ and suggested that IRAS $4 \mathrm{BI}$ is a multiple system. However, later studies could not confirm the multiplicity of BI (Looney et al. 2000; Reipurth et al. 2002). To settle this issue, it is necessary to make sensitive observations with a resolution higher than 0.5 .

While IRAS 4BII has received relatively less attention, it is probably an interesting object in understanding low-mass star formation processes, because its nondetection in infrared suggests that it is an unusually cold object. The lack of detectable star forming activity is also intriguing.

Corresponding Author: M. Choi
In this paper, we present the results of our observations of the NGC 1333 IRAS 4B region in the 6.9 $\mathrm{mm}$ and $1.3 \mathrm{~cm}$ continuum with the Very Large Array (VLA) of the National Radio Astronomy Observatory. We describe our radio continuum observations in Section 2. In Section 3, we report the results of the continuum imaging. In Section 4, we discuss the star-forming activities in the IRAS $4 \mathrm{~B}$ region.

\section{OBSERVATIONS AND DATA}

\subsection{Q-band Observations}

The NGC 1333 IRAS 4B region was observed using VLA in the standard Q-band continuum mode (43.3 $\mathrm{GHz}$ or $\lambda=6.9 \mathrm{~mm}$ ). Twenty-five antennas were used in the C-array configuration on 2004 March 2. The phase-tracking center was $\alpha_{2000}=03^{\mathrm{h}} 29^{\mathrm{m}} 12.988, \delta_{2000}$ $=31^{\circ} 13^{\prime} 08^{\prime \prime} .10$, which is $\sim 2^{\prime \prime}$ east and $\sim 1^{\prime \prime}$ north of IRAS 4BII.

The phase was determined by observing the nearby quasar 0336+323 (PKS 0333+321). The flux was calibrated by setting the flux density of the quasar $0713+438$ (QSO B0710+439) to $0.20 \mathrm{Jy}$, which is the flux density measured within a day of our observations (VLA Calibrator Flux Density Database*). Comparison of the amplitude gave a flux density of $1.36 \mathrm{Jy}$ for $0336+323$. To avoid degradation of sensitivity due to pointing errors, pointing was referenced by observing the calibrators in the $\mathrm{X}$ band $(\lambda=3.6 \mathrm{~cm})$. This referenced pointing was performed about once an hour and just before observing the flux calibrator.

\footnotetext{
* See http://aips2.nrao.edu/vla/calflux.html.
} 


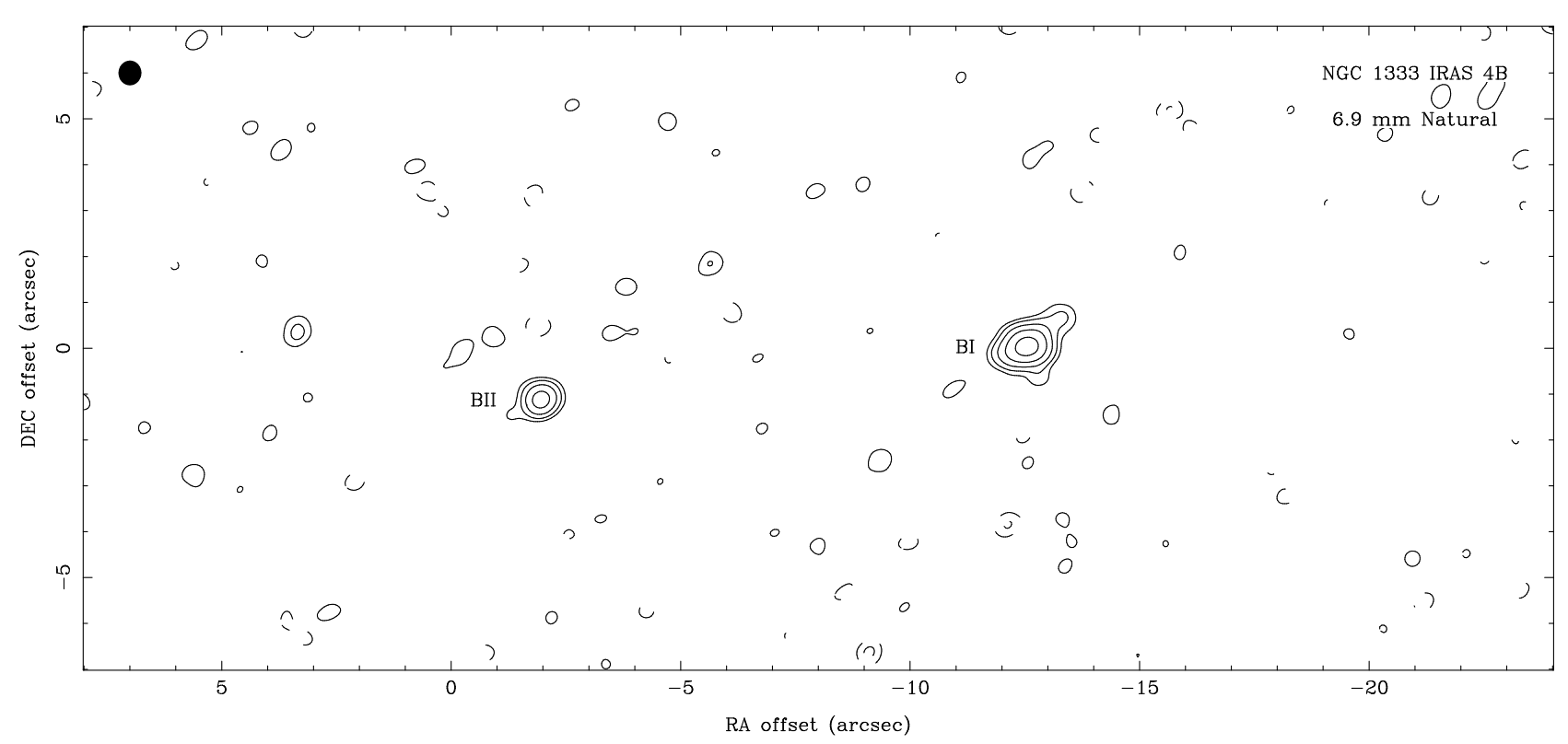

Fig. 1. - Map of the $\lambda=6.9 \mathrm{~mm}$ continuum emission toward the NGC 1333 IRAS $4 \mathrm{~B}$ region, made with a natural weighting. The contour levels are $1,2,4,8$, and $16 \times 0.07 \mathrm{mJy}_{\text {beam }}{ }^{-1}$. Dashed contours are for negative levels. The rms noise is $0.024 \mathrm{mJy}_{\text {beam }}{ }^{-1}$. Shown in the top left-hand corner is the synthesized beam: FWHM $=0^{\prime \prime} .53 \times 0$. .'51 with PA $=$ $-1^{\circ}$. The coordinates are position offsets relative to the phase tracking center of the Q-band observations.

Maps were made using a CLEAN algorithm. With a natural weighting, the $6.9 \mathrm{~mm}$ continuum data produced a synthesized beam of $\sim 0^{\prime \prime} .5$ in full-width at halfmaximum (FWHM).

\section{$2.2 \quad$ K-band Data}

The NGC 1333 IRAS 4 region was observed using VLA in the $\lambda=1.3 \mathrm{~cm}$ continuum. Details of the observations and the results for the IRAS $4 \mathrm{~A}$ region were presented in Choi et al. $(2010,2011)$. The observations were made in the standard K-band continuum mode $(22.5 \mathrm{GHz})$ in the B-array configuration. With a natural weighting, the $1.3 \mathrm{~cm}$ continuum data produced a synthesized beam of FWHM $\approx 0$ ". 4 .

\section{RESULTS}

Fig. 1 shows the $6.9 \mathrm{~mm}$ continuum map toward the NGC 1333 IRAS 4B region. IRAS 4BI and BII were clearly detected. Table 1 lists the continuum source parameters. Fig. 2 shows a map of the BI region with a smaller beam. Fig. 3 shows the same region in the $1.3 \mathrm{~cm}$ continuum. IRAS 4BII was not detected in the $1.3 \mathrm{~cm}$ continuum.

In the $6.9 \mathrm{~mm}$ map (Fig. 2), IRAS 4BI seems to be elongated in the northwest-southeast direction. An elliptical Gaussian fit gives a deconvolved source size of FWHM $=00^{\prime \prime} 56 \times 0^{\prime \prime} 15$ with a position angle $(\mathrm{PA})$ of $-73^{\circ}$. Comparison with the distribution of the $\mathrm{H}_{2} \mathrm{O}$ maser sources (Rodríguez et al. 2002; Park \& Choi 2007 ) suggests that the $6.9 \mathrm{~mm}$ continuum source struc-

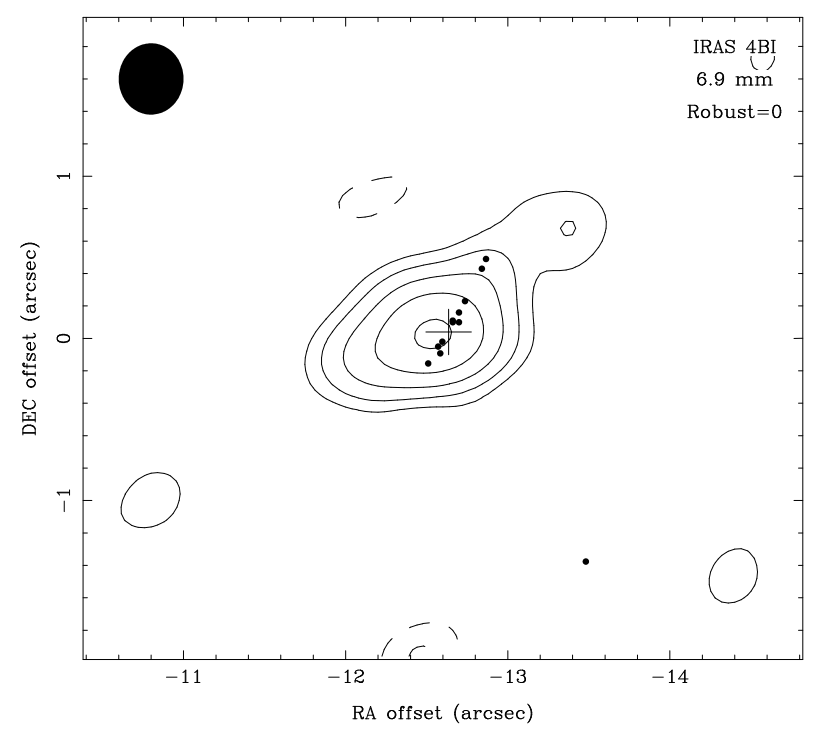

Fig. 2.- Map of the $\lambda=6.9 \mathrm{~mm}$ continuum emission toward the IRAS 4BI region, made with a robust weighting. The contour levels are $1,2,4,8$, and $16 \times 0.07$ mJy beam $^{-1}$. The rms noise is $0.024 \mathrm{mJy}_{\text {beam }^{-1}}$. Shown in the top left-hand corner is the synthesized beam: FWHM $=0$ ". 45 $\times 0^{\prime \prime} .40$ with PA $=-1^{\circ}$. Solid dots: $\mathrm{H}_{2} \mathrm{O}$ maser sources (Rodríguez et al. 2002; Park \& Choi 2007). Plus sign: the $3.6 \mathrm{~cm}$ continuum source (Reipurth et al. 2002).

ture is affected by the outflow, at least in the northwestern part of the source. Possible explanations include shock-heated dusts around the outflow and free- 
Table 1.

NGC 1333 IRAS 4B Continuum Source Parameters

\begin{tabular}{|c|c|c|c|c|c|c|}
\hline \multirow[b]{2}{*}{ Source } & \multicolumn{2}{|c|}{ Peak Position $^{a}$} & \multicolumn{2}{|c|}{$6.9 \mathrm{~mm}$ Flux Density ${ }^{b}$} & \multicolumn{2}{|c|}{$1.3 \mathrm{~cm}$ Flux Density ${ }^{b}$} \\
\hline & $\alpha_{\mathrm{J} 2000.0}$ & $\delta_{\mathrm{J} 2000.0}$ & Peak & Total & Peak & Total \\
\hline$\overline{\mathrm{BI}}$ & 032912.009 & 311308.14 & $1.77 \pm 0.03$ & $3.08 \pm 0.10$ & $0.362 \pm 0.019$ & $0.47 \pm 0.05$ \\
\hline BII & 032912.835 & 311306.98 & $0.77 \pm 0.02$ & $0.89 \pm 0.05$ & $\ldots$ & $\ldots$ \\
\hline
\end{tabular}

a Source positions from the robust-weight $6.9 \mathrm{~mm}$ map (Fig. 2). The $1.3 \mathrm{~cm}$ position of BI agrees with the $6.9 \mathrm{~mm}$ position within $0 . \prime 09$. Units of right ascension are hours, minutes, and seconds, and units of declination are degrees, arcminutes, and arcseconds.

${ }^{b}$ Flux densities are in m.Jy beam ${ }^{-1}$ or $\mathrm{mJy}$, corrected for the primary beam response.

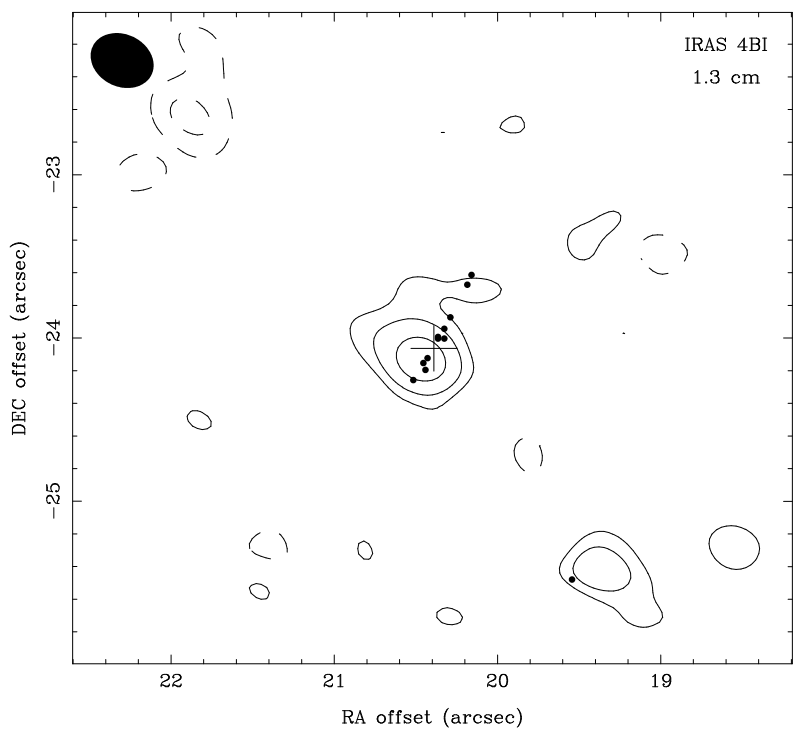

Fig. 3.- Map of the $\lambda=1.3 \mathrm{~cm}$ continuum toward the IRAS 4BI region. The contour levels are 1,2 , and $4 \times$ $0.05 \mathrm{mJy} \mathrm{beam}^{-1}$. The rms noise is $0.016 \mathrm{mJy} \mathrm{beam}^{-1}$. Shown in the top left-hand corner is the synthesized beam: $\mathrm{FWHM}=0$. . $40 \times 0^{\prime \prime} .33$ with $\mathrm{PA}=64^{\circ}$. The coordinates are position offsets relative to the phase tracking center of the K-band observations.

free emission from ionized outflowing gas.

In the $1.3 \mathrm{~cm}$ map (Fig. 3), IRAS 4BI seems to be elongated in the northeast-southwest direction. An elliptical Gaussian fit gives FWHM $=0$ ' $20 \times 0$ "' 10 with $\mathrm{PA}=50^{\circ}$. The size corresponds to $47 \times 24 \mathrm{AU}^{2}$ at a distance of $235 \mathrm{pc}$. Since the elongation is nearly perpendicular to the direction of the $\mathrm{H}_{2} \mathrm{O}$ outflow (PA $\approx 151^{\circ}$ ), the $1.3 \mathrm{~cm}$ continuum may be tracing either a circumstellar disk or a flattened protostellar envelope (pseudodisk), or probably a combination of them.

The $6.9 \mathrm{~mm}$ continuum source of IRAS 4BII (Figure 1) is essentially unresolved. BII is very compact and has never been clearly resolved in interferometric images (e.g., Looney et al. 2000; note that IRAS 4BII was referred to as IRAS $4 \mathrm{C}$ by Looney et al.).

The total flux densities from our data (Table 1) are smaller than those given by Di Francesco et al. (2001) by a factor of $\sim 2.5$. One possible reason for the difference is that our observations were made in more extended array configurations. Another reason could be that the flux calibrator in our observations is different from that used by Di Francesco et al. (2001).

\section{DISCUSSION}

\subsection{IRAS 4BI}

\subsubsection{Source Structure}

The single-baseline submillimeter interferometric observations of Lay et al. (1995) suggested that the source structure of IRAS 4BI cannot be explained as a single source. Even a binary model could not fit the data well, and they suggested that BI is a triple or higher-order multiple system. Considering their resolution and the clear dips in the visibility curve, BI was expected to consist of multiple components of comparable brightness with a separation larger than $00^{\prime \prime} 5$. In later studies with resolutions higher than $1^{\prime \prime}$, however, the image of IRAS 4BI was always found to be dominated by a single peak (Looney et al. 2000; Reipurth et al. 2002; Choi et al. 2007; Jørgensen et al. 2007; Jørgensen \& van Dishoeck 2010).

Our $6.9 \mathrm{~mm}$ continuum map (Fig. 2) shows a weak peak located about $1^{\prime \prime}$ northwest of IRS 4BI. This peak is probably not a real source, considering that the map shows some negative peaks at comparable (absolute) intensities and that the $2.7 \mathrm{~mm}$ map of Looney et al. (2000) does not show a corresponding peak. Even if it is real, it could not have caused the interference pattern seen by Lay et al. (1995) because it is weaker than the BI main peak by a factor of $\sim 9$. The elongated shape of the source (Section 3) could have caused some interference pattern. Another possible source of confusion is BII, because it is located in the primary beam of Lay et al. and its existence was not known at the time of their analysis. 


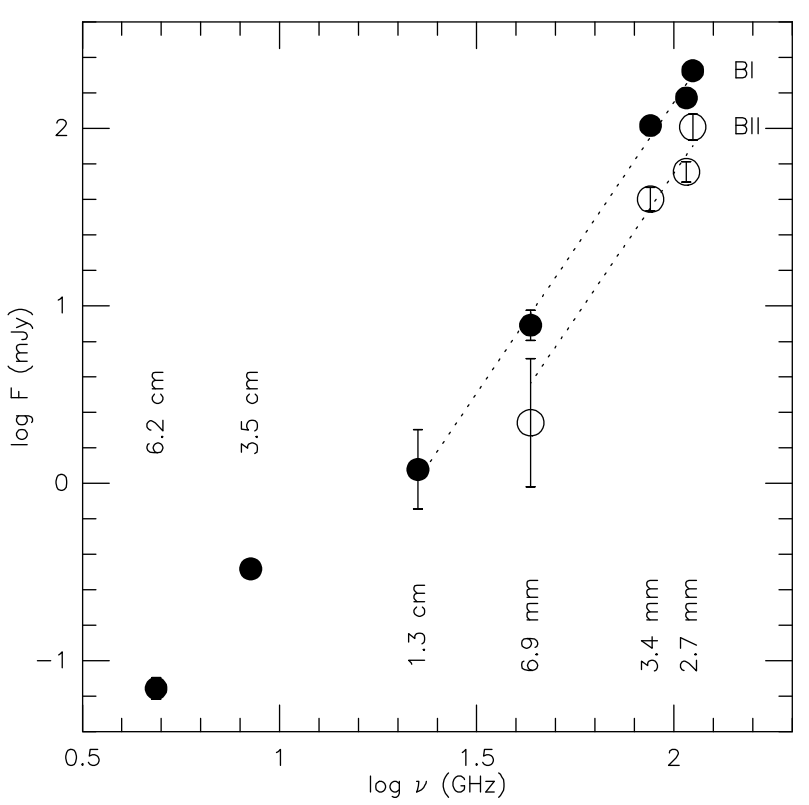

Fig. 4.- Spectral energy distributions of the IRAS 4B sources. Filled circles: BI. Open circles: BII. Flux densities are from Rodríguez et al. (1999), Reipurth et al. (2002), Di Francesco et al. (2001), Choi et al. (2001), and Looney et al. (2000). The flux uncertainties are smaller than the size of markers except for the $1.3 \mathrm{~cm}$ and $6.9 \mathrm{~mm}$ data. Dotted lines: Best-fit power-law spectra in the $(1.3 \mathrm{~cm}, 2.7 \mathrm{~mm})$ section.

\subsubsection{Continuum Spectrum and Mass}

Fig. 4 shows the spectral energy distribution (SED) of IRAS 4BI. (For the SED and mass estimates, we use the flux densities given by Di Francesco et al. (2001), $7.8 \mathrm{mJy}$ at $6.9 \mathrm{~mm}$ and $1.2 \mathrm{mJy}$ at $1.3 \mathrm{~cm}$, because the values in Table 1 might suffer from missing flux.) The steep slope in the $(1.3 \mathrm{~cm}, 2.7 \mathrm{~mm})$ section suggests that the millimeter flux comes from thermal emission of dust. The SED in this section can be described using a power-law form, $F \propto \nu^{\alpha}$, where $F$ is the flux density, $\nu$ is the frequency, and $\alpha$ is the spectral index. The best-fit power-law spectrum gives $\alpha=3.3 \pm 0.2$. The $3.5-6.2 \mathrm{~cm}$ flux may be mostly from free-free emission of a thermal radio jet. The free-free emission seems to be optically thick at $6.2 \mathrm{~cm}$.

The mass of the circumstellar molecular gas can be derived from the dust continuum in the millimeter bands, using the procedure and equations described in Section 5 of Choi (2001) and the mass emissivity given by Beckwith \& Sargent (1991). The best-fit spectrum gives an opacity index of $\beta \approx 1.3$. Assuming a distance of $235 \mathrm{pc}$ to the source and a dust temperature of 33 K (Hirota et al. 2008; Jennings et al. 1987), the mass of IRAS $4 \mathrm{BI}$ is $1.0 \pm 0.5 \mathrm{M}_{\odot}$, which may include the inner protostellar envelope and the disk. For comparison, the mass estimates of BI in previous works (scaled to the distance of $235 \mathrm{pc}$ ) are $0.43 M_{\odot}$ in Looney et al. (2000), $1.1 M_{\odot}$ in Choi (2001), and 0.47/0.26 $M_{\odot}$

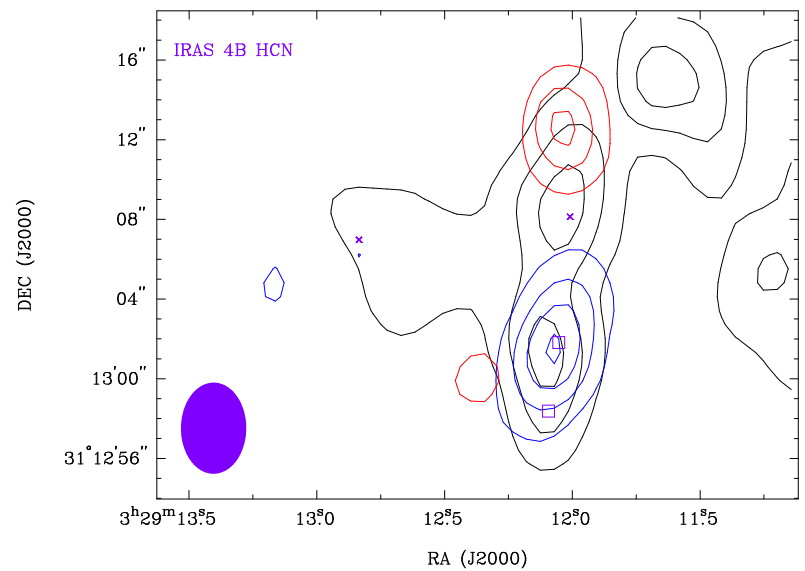

Fig. 5.- Maps of the $\mathrm{HCN} J=1 \rightarrow 0$ line toward the IRAS 4B region, from the data presented by Choi (2001). For the line core of the $F=2 \rightarrow 1$ hyperfine component (black contours), the HCN line was averaged over the $V_{\mathrm{LSR}}$ interval of $5.8-7.2 \mathrm{~km} \mathrm{~s}^{-1}$. The lowest contour level and the contour interval are $150 \mathrm{mJy}_{\text {beam }}^{-1}$, and the rms noise is $50 \mathrm{mJy}$ beam $^{-1}$. For the blueshifted and redshifted outflows (blue and red contours, respectively), see Fig. 1 and 2 of Choi (2001) for the velocity intervals. The lowest contour level and the contour interval are $60 \mathrm{mJy}$ beam $^{-1}$, and the $\mathrm{rms}$ noise is $20 \mathrm{mJy}$ beam $^{-1}$. Shown at the bottom lefthand corner is the synthesized beam: FWHM $=4{ }^{\prime \prime} .6 \times 33^{\prime \prime} 3$ and $\mathrm{PA}=0^{\circ}$. Crosses: the $6.9 \mathrm{~mm}$ continuum sources. Squares: The $\mathrm{H}_{2}$ line peaks HL 9a/b (Choi et al. 2006; Fig. 6a).

in Jørgensen et al. (2007). The mass of the whole protostellar envelope of BI derived from single-dish observations is $\sim 3.2 M_{\odot}$ (Enoch et al. 2009 , scaled to $235 \mathrm{pc})$.

\subsubsection{Outflow and Infrared Images}

IRAS 4BI drives a bipolar outflow roughly in the north-south direction. Interferometric images of several molecular lines show the redshifted lobe to the north and the blueshifted lobe to the south of BI (Fig. 5; Choi 2001; Di Francesco et al. 2001; Jørgensen et al. 2007; Choi et al. 2011). In these millimeter lines the northern and southern lobes are often comparable in brightness. Images of the infrared molecular hydrogen line show a jet-like flow to the south only (Hodapp \& Ladd 1995; Choi et al. 2006). The bright $\mathrm{H}_{2}$ knots are referred to as HL 9a/b (Fig. 6a). The $\mathrm{H}_{2}$ jet may be bipolar in nature, but the unseen northern jet may be obscured by the dense protostellar envelope. Recent far-IR observations showed highly excited $\mathrm{H}_{2} \mathrm{O}$ emission from the southern outflow (Herczeg et al. 2011).

The direction of the outflow, i.e., the position angle of HL 9 with respect to the $6.9 \mathrm{~mm}$ continuum source of IRAS $4 \mathrm{BI}$, is $\sim 174^{\circ}$. By contrast, the position angle of the small-scale jet traced by the $\mathrm{H}_{2} \mathrm{O}$ maser is $\sim 151^{\circ}$ (Rodríguez et al. 2002; Park \& Choi 2007). To explain the difference in the outflow directions, it was proposed 


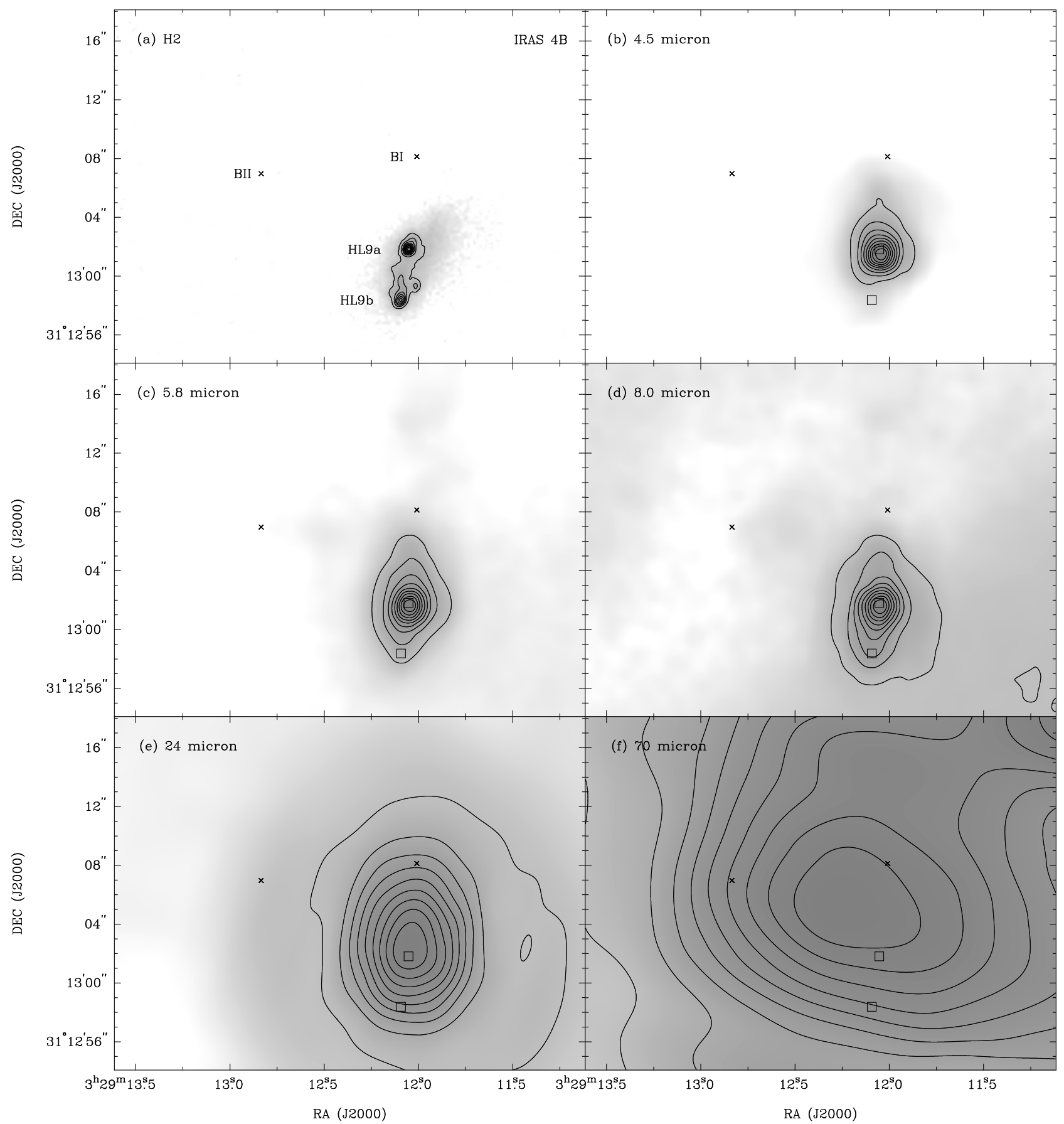

Fig. 6. - IR images of the IRAS $4 \mathrm{~B}$ region. (a) Map of the $\mathrm{H}_{2} 1-0 S(1)$ line. The emission peak positions are $\alpha_{2000}=$ $03^{\mathrm{h}} 29^{\mathrm{m}} 12^{\mathrm{s}} .05, \delta_{2000}=31^{\circ} 13^{\prime} 01^{\prime \prime} .8$ for HL $9 \mathrm{a}$ and $03^{\mathrm{h}} 29^{\mathrm{m}} 12^{\mathrm{s}} .09,31^{\circ} 12^{\prime} 58^{\prime \prime} .4$ for HL $9 \mathrm{~b}$. (b) $4.5 \mu \mathrm{m}$ image. (c) $5.8 \mu \mathrm{m}$ image. (d) $8.0 \mu \mathrm{m}$ image. (e) $24 \mu \mathrm{m}$ image. (f) $70 \mu \mathrm{m}$ image. The lowest contour level and the contour interval are $10 \%$ of the maximum intensity in each image. The highest contour is at the $90 \%$ level. The grayscale images are in a logarithmic scale and show low-level features. The $\mathrm{H}_{2}$ image is from the Subaru telescope (see Fig. 2 of Choi et al. 2006), and the others are from the Spitzer data archive.

that the jet is precessing at a high rate (Marvel et al. 2008; Desmurs et al. 2009). (Marvel et al. (2008) also suggested that there are probably two outflows, i.e., BI may be a binary system.) The position angles of HL 9a and $9 \mathrm{~b}$ with respect to $\mathrm{BI}$ agree within $\sim 1^{\circ}$ and show no sign of precession to the proposed direction. 
In the infrared images of the IRAS $4 \mathrm{~B}$ region, the $\mathrm{H}_{2}$ jet, especially the bright knot HL 9a, is the dominating emission feature. In the Spitzer images, only HL 9a/b can be seen in the 3.6-24 $\mu \mathrm{m}$ bands (Fig. 6b-e), and neither BI nor BII is clearly detectable. Even at the $70 \mu \mathrm{m}$ band, the peak position is located between BI and HL 9a (Fig. 6f), and the interpretation of the image and the flux is ambiguous. Note that the Spitzer source SSTc2dJ032912.04+311301.5 corresponds to HL 9a, and the SED of this source (Per-emb 13) presented by Enoch et al. (2009) is a combination of the flux densities of HL 9a on the short-wavelength side and those of BI on the long-wavelength side. Therefore, their bolometric temperature and luminosity of the BI protostar may be overestimated. This example suggests that, at least for some young stellar objects, the emission from secondary sources (such as outflows and/or binary companion) can "contaminate" the infrared flux and make the SED appear hotter. Such effects can introduce a bias into SED surveys of young stellar objects.

\subsection{IRAS $4 \mathrm{BII}$}

IRAS 4BII has been detected as a compact source in interferometric images of millimeter and submillimeter continuum (Looney et al. 2000; Choi 2001; Di Francesco et al. 2001; Jørgensen et al. 2007; Jørgensen \& van Dishoeck 2010) and in a single-dish submillimeter-continuum map (Smith et al. 2000). However, its evolutionary status and star-formation activity are not well known. Single-dish radio observations usually do not have angular resolutions high enough to separate BII from BI, and BII has never been detected in infrared. In molecular lines, BII was detected in the ${ }^{13} \mathrm{CO}$ and $\mathrm{HCN}$ line maps only (Fig. 5; Choi 2001). There is no known molecular outflow associated with BII. The nondetection in the centimeter continuum (Rodríguez et al. 1999; Reipurth et al. 2002) also suggests that there is no detectable radio jet. Since there is no detection in infrared, the SED of $\mathrm{BII}$ is incomplete and cannot be classified well enough to tell the evolutionary status.

The best-fit millimeter SED of IRAS 4BII (Figure 4) gives a mass of $\sim 0.4 M_{\odot}$, using the same procedure and assumptions in Section 4.1.2. The mass estimate, however, is very uncertain because the $6.9 \mathrm{~mm}$ flux density (hence the opacity index) has a relatively large uncertainty. The uncertainty interval of the mass estimate is $0.1-2.3 M_{\odot}$. For comparison, the mass estimates of BII in previous works (scaled to the distance of 235 pc) are $0.12 M_{\odot}$ in Looney et al. (2000), $2.2 M_{\odot}$ in Choi (2001), and $0.17 / 0.074 M_{\odot}$ in Jørgensen et al. (2007). Note that the dust temperature of $33 \mathrm{~K}$ used in the mass estimate came from the SED of the whole IRAS 4 system (Jennings et al. 1987). Since BII is undetectable in infrared, it can be much colder, and the mass can be larger than the values given above.

On the nature of IRAS 4BII, previous studies sug- gested that it may be an optical/IR source (such as T Tauri star) or a Class I young stellar object (Looney et al. 2000; Choi 2001). The nondetection of BII in the Spitzer images (Fig. 6), however, seems to rule out these possibilities. One possible explanation (that was not known at the time of the previous studies) is that it can be a newly recognized type of young stellar objects known as very low luminosity objects (VeLLOs; Young et al. 2004; Crapsi et al. 2005; Lee 2007; Dunham et al. 2008). The internal luminosity of a VeLLO is less than $0.1 L_{\odot}$, which is much lower than what is predicted by models of low-mass star formation (Shu et al. 1987).

Dunham et al. (2008) found that the $70 \mu \mathrm{m}$ flux correlates well with the internal luminosity. Assuming that the strong source in the $70 \mu \mathrm{m}$ image (Fig. 6f) is IRAS 4BI, the image was fitted with a point source. The residual map gives an upper limit of $\sim 500 \mathrm{mJy}$ on the flux density of BII. Using the scaling given by Dunham et al. (2008), the upper limit on the internal luminosity of BII is $\sim 0.08 L_{\odot}$. Therefore, it is possible that BII may be a VeLLO.

The compact structure of IRAS 4BII and the nondetection of any star-formation activity point to yet another possibility: the first hydrostatic core (FHSC; Boss \& Yorke 1995; Masunaga et al. 1998). Since the observational characteristics of FHSC have not been well established yet (e.g., Enoch et al. 2010), it is not clear if BII is such an object. More sensitive and higherresolution observations in the far-IR band are needed to verify the status of BII as VeLLO, FHSC, or any other possibilities.

\section{ACKNOWLEDGMENTS}

We thank Miju Kang and Gregory J. Herczeg for helpful discussions and suggestions. M. C. was supported by the Core Research Program of the National Research Foundation of Korea (NRF) funded by the Ministry of Education, Science and Technology (MEST) of the Korean government (grant number 2011-0015816). J.-E. L. was supported by the Basic Science Research Program through NRF funded by MEST (grant number 2011-0004781). The National Radio Astronomy Observatory is a facility of the National Science Foundation operated under cooperative agreement by Associated Universities, Inc. This work is based in part on observations made with the Spitzer Space Telescope, which is operated by the Jet Propulsion Laboratory, California Institute of Technology, under a contract with NASA.

\section{REFERENCES}

Beckwith, S. V. W., \& Sargent, A. I. 1991, Particle Emissivity in Circumstellar Disks, ApJ, 381, 250

Blake, G. A., Sandell, G., van Dishoeck, E. F., Groesbeck, T. D., Mundy, L. G., \& Aspin, C. 1995, A Molecular Line Study of NGC 1333/IRAS 4, ApJ, 441,689 
Boss, A. P., \& Yorke, H. W. 1995, Spectral Energy of First Protostellar Cores: Detecting "Class -I" Protostars with ISO and SIRTF, ApJ, 439, L55

Choi, M. 2001, High-Resolution Observations of the Molecular Clouds in the NGC 1333 IRAS 4 Region, ApJ, 553, 219

Choi, M., Hodapp, K. W., Hayashi, M., Motohara, K., Pak, S., \& Pyo, T.-S. 2006, Variability of the NGC 1333 IRAS 4A Outflow: Molecular Hydrogen and Silicon Monoxide Images, ApJ, 646, 1050

Choi, M., Kamazaki, T., Tatematsu, K., \& Panis, J.-F. 2004, Structure of the Dense Molecular Gas in the NGC 1333 IRAS 4 Region, ApJ, 617, 1157

Choi, M., Kang, M., Tatematsu, K., Lee, J.-E., \& Park, G. 2011, Radio Imaging of the NGC 1333 IRAS 4A Region: Envelope, Disks, and Outflows of a Protostellar Binary System, PASJ, 63, in press (arXiv:1107.3877)

Choi, M., Panis, J.-F., \& Evans, N. J., II 1999, Berkeley-Illinois-Maryland Association Survey of Protostellar Collapse Candidates in $\mathrm{HCO}^{+}$and HCN Lines, ApJS, 122, 519

Choi, M., Tatematsu, K., \& Kang, M. 2010, Kinematics of the Ammonia Disk around the Protostar NGC 1333 IRAS 4A2, ApJ, 723, L34

Choi, M., Tatematsu, K., Park, G., \& Kang, M. 2007, Ammonia Imaging of the Disks in the NGC 1333 IRAS 4A Protobinary System, ApJ, 667, L183

Crapsi, A., et al. 2005, Dynamical and Chemical Properties of the "Starless" Core L1014, A\&A, 439, 1023

Desmurs, J.-F., Codella, C., Santiago-García, J., Tafalla, M., \& Bachiller, R. 2009, The Extremely Collimated Bipolar $\mathrm{H}_{2} \mathrm{O}$ Jet from the NGC 1333IRAS 4B Protostar, A\&A, 498, 753

Di Francesco, J., Myers, P. C., Wilner, D. J., Ohashi, N., \& Mardones, D. 2001, Infall, Outflow, Rotation, and Turbulent Motions of Dense Gas within NGC 1333 IRAS 4, ApJ, 562, 770

Dunham, M. M., Crapsi, A., Evans, N. J., II, Bourke, T. L., Huard, T. L., Myers, P. C., \& Kauffmann, J. 2008, Identifying the Low-Luminosity Population of Embedded Protostars in the c2d Observations of Clouds and Cores, ApJS, 179, 249

Enoch, M. L., Evans, N. J., II, Sargent, A. I., \& Glenn, J. 2009, Properties of the Youngest Protostars in Perseus, Serpens, and Ophiuchus, ApJ, 692, 973

Enoch, M. L., Lee, J.-E., Harvey, P., Dunham, M. M., \& Schnee, S. 2010, A Candidate Detection of the First Hydrostatic Core, ApJ, 722, L33

Herczeg, G. J., et al. 2011, Water in Star-Forming Regions with Herschel: Highly Excited Molecular Emission from the NGC 1333 IRAS4B Outflow, submitted to A\&A
Hirota, T., et al. 2008, Astrometry of $\mathrm{H}_{2} \mathrm{O}$ Masers in Nearby Star-Forming Regions with VERA. II. SVS13 in NGC1333, PASJ, 60, 37

Hodapp, K.-W., \& Ladd, E. F. 1995, Bipolar Jets from Extremely Young Stars Observed in Molecular Hydrogen Emission, ApJ, 453, 715

Jennings, R. E., Cameron, D. H. M., Cudlip, W., \& Hirst, C. J. 1987, IRAS Observations of NGC1333, MNRAS, 226, 461

Jørgensen, J. K., \& van Dishoeck, E. F. 2010, Water Vapor in the Inner $25 \mathrm{AU}$ of a Young Disk around a Low-Mass Protostar, ApJ, 710, L72

Jørgensen, J. K., et al. 2007, PROSAC: A Submillimeter Array Survey of Low-Mass Protostars. I. Overview of Program: Envelopes, Disks, Outflows, and Hot Cores, ApJ, 659, 479

Lay, O. P., Carlstrom, J. E., \& Hills, R. E. 1995, NGC 1333 IRAS 4: Further Multiplicity Revealed with the CSO-JCMT Interferometer, ApJ, 452, L73

Lee, J.-E. 2007, Chemical Evolution in VeLLOs, JKAS, 40,85

Looney, L. W., Mundy, L. G., \& Welch, W. J. 2000, Unveiling the Circumstellar Envelope and Disk: A Subarcsecond Survey of Circumstellar Structures, ApJ, 529,477

Marvel, K. B., Wilking, B. A., Claussen, M. J., \& Wootten, A. 2008, Time-Resolved AU-Scale Jets Traced by Masers in the IRAS 4A/B Regions of NGC 1333, ApJ, 685, 285

Masunaga, H., Miyama, S. M., \& Inutsuka, S.-I. 1998, A Radiation Hydrodynamic Model for Protostellar Collapse. I. The First Collapse, ApJ, 495, 346

Park, G., \& Choi, M. 2007, Observations of Water Masers in the NGC 1333 IRAS 4 Region, ApJ, 664, L99

Reipurth, B., Rodríguez, L. F., Anglada, G., \& Bally, J. 2002, Radio Continuum Maps of Deeply Embedded Protostars: Thermal Jets, Multiplicity, and Variability, AJ, 124, 1045

Rodríguez, L. F., Anglada, G., \& Curiel, S. 1999, The Nature of the Radio Continuum Sources Embedded in the HH 7-11 Region and Its Surroundings, ApJS, 125,427

Rodríguez, L. F., Anglada, G., Torrelles, J. M., Mendoza-Torres, J. E., Haschick, A. D., \& Ho, P. T. P. 2002, VLA Observations of Water Maser Emission Associated with SVS 13 and Other Sources in NGC 1333, A\&A, 389, 572

Sandell, G., Aspin, C., Duncan, W. D., Russell, A. P. G., \& Robson, E. I. 1991, NGC 1333 IRAS 4 - A Very Young, Low-Luminosity Binary System, ApJ, 376, L17

Shu, F. H., Adams, F. C., \& Lizano, S. 1987, Star Formation in Molecular Clouds: Observation and Theory, ARA\&A, 25, 23 
Smith, K. W., Bonnell, I. A., Emerson, J. P., \& Jenness, T. 2000, NGC 1333/IRAS 4: a Multiple Star Formation Laboratory, MNRAS, 319, 991

Young, C. H., et al. 2004, A "Starless" Core that Isn't: Detection of a Source in the L1014 Dense Core with the Spitzer Space Telescope, ApJS, 154, 396 